\title{
Diagnostic Value of Serum Biomarker Human Epididymis Protein4 in Ovarian Cancers
}

\author{
Rimaz Elhag Gurashi ${ }^{1}$, Moawia Elsadig Hummeida ${ }^{2}$, Faisal Galal Abdelaziz ${ }^{3}$ \\ ${ }^{1}$ Clinical Chemistry Department, Faculty of Medical Laboratory Sciences, Al-neelain University, Khartoum, Sudan \\ ${ }^{2}$ Department of Obgyn, Faculty of Medicine, Al-neelain University, Khartoum, Sudan \\ ${ }^{3}$ Gynological Oncologist, Military Hospital, Omdurman, Sudan
}

Email address:

rimazgourashi@gmail.com (R. E. Gurashi)

\section{To cite this article:}

Rimaz Elhag Gurashi, Moawia Elsadig Hummeida, Faisal Galal Abdelaziz. Diagnostic Value of Serum Biomarker Human Epididymis Protein4 in Ovarian Cancers. American Journal of Laboratory Medicine. Vol. 5, No. 1, 2020, pp. 18-27. doi: 10.11648/j.ajlm.20200501.13

Received: October 6, 2019; Accepted: December 27, 2019; Published: January 17, 2020

\begin{abstract}
Ovarian cancer is the fifth most common cancer affecting women today. In fact, ovarian cancer is responsible for more deaths than any other type of female reproductive cancer. According to the American Cancer Society, 20,000 women are diagnosed with ovarian cancer each year. Since diagnosis early on is associated with improved rates of survival, an effective screening strategy that detects early stage ovarian cancer may have a significant impact on mortality from the disease. Cancer researchers are under way to develop more accurate tumor markers that can be used to identify disease in its early stages, to predict the effectiveness of treatment, or to predict the chance of cancer recurrence after treatment has ended. Cancer antigen 125 (CA125) is an established biomarker for ovarian cancer detection. As CA125 effectiveness in the identification of the malignancy is threatened by its low diagnostic specificity, measurements of other tumor marker, Human epididymis protein 4 (He4), in the serum have been proposed for improving the sensitivity and specificity of laboratory identification of the disease. The aim of our research study was to evaluate the diagnostic performance of Human epididymis protein4 (He4) in ovarian cancer patients. Our study group consisted of 90 Sudanese ladies age range (16-80) years old attending Gynological Oncology clinics in Omdurman Military hospitals. Blood samples were collected and centrifugated using standardized procedure, all analyses were performed in serum samples. Human epididymis protein 4 (HE4) serum concentration were determined using Enzyme-Linked Immunosorbent Assay (CUSABIO ELISA kits) for the quantitative invitro diagnostic measurement. the data were treated Statistically. The study results shown that epithelial ovarian cancer is the most common ovarian cancer type in Sudan followed by germ cell tumors. Serum level of Human epididymis protein 4 (HE4) biomarker within the reference range in the control group. In contrast, increasing serum level of Human epididymis protein 4 (HE4) were obtained in the ovarian cancer patients, A general agreement that a combination of multiple biomarkers may increase diagnostic sensitivity and specificity over use of individual marker. The results of this study confirmed that, by combining He4 measurements with cancer antigen 125, we can improve the diagnostics performance for OC. HE4 is a relatively stable serum biomarker for ovarian cancer with a higher diagnostic prediction.
\end{abstract}

Keywords: Ovarian Cancer, Biomarker, HE4, Epithelial OV

\section{Introduction and Literature Review}

Ovarian cancer is a malignant tumour in one or both ovaries. It can start in any of the three cell types found in the ovary Epithelial ovarian, fallopian tube and peritoneal cancers all develop in the same type of cell and are very similar Recent research suggests that many epithelial ovarian cancers start in the fallopian tubes. It has been called the "silent killer" because symptoms often become apparent only when the cancer has spread and is harder to treat. It's the fifth leading cause of cancer-related death in women in the United States and is the leading cause of gynecologic cancer deaths. Despite being one-tenth as common as breast cancer, it is three times more lethal, and carries a 1:70 lifetime risk. This year, approximately 20,180 women will be diagnosed with ovarian cancer, and 15,310 will die in USA from the disease [1]. The high mortality rate of ovarian cancer is due to the 
lack of a screening strategy to detect early-stage disease. Ovarian cancer presents with very few, if any, specific symptoms [2].

Over the past quarter of a century, several scientific developments have challenged traditional concepts in ovarian cancer. First, it was recognized that ovarian cancer is not a homogeneous disease, but rather a group of diseases-each with different morphology and biological behavior. Approximately $90 \%$ of ovarian cancers are carcinomas and, based on histopathology, immunohistochemistry, and molecular genetic analysis, at least five main types are currently distinguished: high-grade serous carcinoma (HGSC, 70\%); endometrioid carcinoma (EC, 10\%); clear-cell carcinoma (CCC, 10\%); mucinous carcinoma (MC, 3\%); and low-grade serous carcinoma (LGSC, <5\%) [3, 4]. These tumor types (which account for $98 \%$ of ovarian carcinomas) can be reproducibly diagnosed by light microscopy and are inherently different diseases, as indicated by differences in epidemiologic and genetic risk factors; precursor lesions; patterns of spread; and molecular events during oncogenesis, response to chemotherapy, and prognosis [3, 4]. Much less common are malignant germ cell tumors and potentially malignant sex cord-stromal tumors. The biomarker expression profile within a given histotype is consistent across stages. Ovarian cancers differ primarily based on histologic type $[5,6]$.

The International Federation of Gynecology and Obstetrics in US (FIGO) stages ovarian tumors on a scale of I to IV according to how well- or poorly-organized the tumors are and whether the cancer is metastasized. Stage I is cancer that is localized and contained in the ovary or ovaries. Stage II is cancer that has spread to other pelvic organs such as the uterus, bladder, or rectum, but is confined to the pelvis [6]. Stage III is cancer that has spread to the lymph nodes and/or abdominal lining and organs, with possible superficial liver metastases. Stage IV is cancer that has spread to distant organs, such as the brain, bone, lungs, or liver parenchyma [ 6 , 7].

Risk Factors for Ovarian Cancer according to American Joint Committee on Cancer 2012 and American Cancer Society 2016, there are several specific factors that change a woman's likelihood of developing ovarian cancer include:

Age, the risk of developing ovarian cancer gets higher with age and, rare in women younger than 40. Most ovarian cancers develop after menopause. Half of all ovarian cancers are found in women 63 years of age or older [8]. Obesity, Melinda $M$ and coauthors [10] have looked at the relationship of obesity and ovarian cancer. Overall $[8,9]$, it seems that obese women have a higher risk of developing ovarian cancer and obesity is associated with a weak adverse effect on the survival of women with ovarian cancer [10].

Reproductive history, several studies have suggested that the ovarian cancer risk reductions associated with parity and oral contraceptive use are weaker in postmenopausal than premenopausal women; yet little is known about the persistence of these reductions as women age. This question gains importance with the increasing numbers of older ovarian cancer women. parity women have a lower risk of ovarian cancer than nulliparity. The risk goes down with each full-term pregnancy and, women who have their first fullterm pregnancy after age 35 or nulliparity have a higher risk of ovarian cancer [11].

Breastfeeding, the evidence that breastfeeding protects against ovarian cancer is well established epidemiologically, recent evidence finds a $37 \%$ reduction for ovarian cancer for women who have breastfed for a year or more [12]. Reduced risk of ovarian cancers related to prolong periods of time during which women do not ovulate or have their menstrual cycles. Later onset of puberty and first menstrual cycles, and an earlier menopause, both of which mean fewer lifetime ovulatory cycles, are associated with decreased risk of ovarian cancer. Contraceptive, women who have used oral and an injectable contraceptive have a lower risk of ovarian cancer. and the risk is lower the longer the contraceptives are used [13]. Gynecologic surgery, tubal ligation may reduce the chance of developing ovarian cancer by up to two-thirds and, hysterectomy also seems to reduce the risk of getting ovarian cancer by about one-third [11]. Fertility drugs, researchers have found that using the fertility drug for longer than one year may increase the risk for developing ovarian tumors, the risk seemed to be increase the risk of low malignant potential ovarian tumor [10].

Estrogen therapy and hormone therapy, recent studies done by Muhammad Zahid eta 1 [14], suggest women using estrogens after menopause have an increased risk of developing ovarian cancer for at least 5 years, the increased risk is less certain for women taking both estrogen and progesterone [14].

About 5 to $10 \%$ of ovarian cancers are a part of family cancer syndromes resulting from inherited mutations in certain genes like what happened in hereditary breast and ovarian cancer syndrome, this syndrome is caused by inherited mutations in the genes BRCA1 and BRCA2, these genes are tumor suppressor genes involved in the regulation of cellular proliferation, chromosomal stability, and DNA repair which linked to a high risk of breast cancer as well as ovarian, fallopian tube, primary peritoneal cancers, pancreatic cancer and prostate cancer, are also increased [15]. According to American collage of Obstetrician and Gynecologist in 2017 [16] the lifetime ovarian cancer risk for women with a BRCA1 mutation is estimated to be between $35 \%$ and $70 \%$. For women with BRCA2 mutations the risk has been estimated to be between $10 \%$ and $30 \%$ by age 70 . These mutations also increase the risks for primary peritoneal carcinoma and fallopian tube carcinoma. In comparison, the ovarian cancer lifetime risk for the women in the general population is less than $2 \%$ in USA [16].

PTEN tumor hamartoma syndrome (Cowden disease) people are primarily affected with thyroid problems, thyroid cancer, and breast cancer. Women also have an increased risk of ovarian cancer. It is caused by inherited mutations in the PTEN gene. Women with Hereditary nonpolyposis colon cancer (Lynch syndrome) have a very high risk of colon cancer and also have an increased risk of developing of 
ovarian and endometrial cancer and many different genes include MLH1, MLH3, MSH2, MSH6, TGFBR2, PMS1, and PMS2 which reduces ability to repair damage to its DNA. The lifetime risk of ovarian cancer in women with hereditary nonpolyposis colon cancer (HNPCC) is about $10 \%$. Up to $1 \%$ of all ovarian epithelial cancers occur in women with this syndrome [16].

Peutz-Jeghers syndrome is a rare genetic syndrome caused by STK11 gene mutations this syndrome develops polyps in the stomach and intestine in teenagers. Women with this syndrome have an increased risk of both epithelial ovarian cancer and sex cord tumor with annular tubules (SCTAT). Personal history of breast cancer has an increased risk of developing ovarian cancer, because one subtype of breast cancer shares many genetic features with high-grade serous ovarian cancer, a cancer that is very difficult to treat, according to researchers supported by the National Institutes of Health [17]. The findings suggest that the two cancers are of similar molecular origin, which may facilitate the comparison of therapeutic data for subtypes of breast and ovarian cancers [17].

There are many lowering ovarian cancer risk factors including, history of pregnancy has a 50\% lower risk of ovarian cancer than women who were never pregnant (nulliparous), and a protective effect is shown in women with multiple pregnancies, oral contraceptive, tubal ligation and hysterectomy also have been associated with a reduced risk of ovarian cancer [18].

Causes Ovarian Cancer, there are many theories about the causes of ovarian cancer, can be classified to exogenous and endogenous factors. The exogenous factors including, Estrogen therapy and hormone therapy, smoking and alcohol induced, exposure to carcinoids materials and radiation, diet with heavy fatty and proceeding meat [10]. Endogenous factors, the hormonal imbalance is important causes of ovarian cancer because it's hormonal dependent cancer, also researchers find a relationship between ovulation and the risk of developing ovarian cancer [15, 16]. Genetic mutations either inherited mutations in the BRCA1 and BRCA2 genes, as well as the genes related to other family cancer syndromes linked to an increased risk of ovarian cancer, such as PTEN tumor hamartoma syndrome, Peutz-Jeghers syndrome, MUTYH-associated polyposis, and the many genes that can cause hereditary nonpolyposis colon cancer (MLH1, MLH3, MSH2, MSH6, TGFBR2, PMS1, and PMS2). or acquired like the TP53 tumor suppressor gene or the HER2 oncogene mutation.

Incidence and Prevalence, according to American cancer society an estimated 22,400 new cases of ovarian cancer in 2017 and about 14,080 deaths will occur in 2017, accounts for $5 \%$ of all cancers in women [20], and A total of 7,378 new cases were reported in the UK in 2014 and it has the highest mortality of all gynecological cancers, accounting for $6 \%$ of all cancer deaths in women [19] Although ovarian cancer occurs most commonly after menopause (average age is 63), it may develop at any age. A woman's risk of developing ovarian cancer in her lifetime is 1 in 71 , and her risk of dying from the disease 1 in 95 . The 5 years survival rate for ovarian cancer is relatively low $(46 \%)$ because most patients are diagnosed with distance stage disease, for which survival is $29 \%$. Survival also varies subsequently by age, with women younger than 45 much more likely to survive 5 years than women 75 and older (77\% versus $20 \%$ ) [20].

Diagnosis of ovarian cancer history, is nonspecific in that symptoms in early-stage disease are either absent or vague and may resemble menopausal symptoms and intestinal illnesses. Individuals in later stages may report indigestion, gas, nausea, vomiting, loss of appetite, a feeling of fullness after small meals, pelvic or abdominal pain, swelling, increased frequency or urgency of urination, unexplained change in bowel habits, unexplained weight gain or loss, pain during intercourse, ongoing fatigue, lower back pain, shortness of breath, and, in rare cases, postmenopausal vaginal bleeding. These symptoms usually do not become apparent until the later stages of the disease when the cancer mass is large enough to interfere with pelvic organs such as the bladder or rectum, or after the cancer has metastasized to the abdominal cavity. Obtaining a personal obstetric and gynecologic history and a family history of gynecologic disease may be important in diagnosis [21]. A number of case-control studies investigating symptoms in women with ovarian cancer and comparing them to symptoms in women without ovarian cancer demonstrate that patients with ovarian cancer are symptomatic for a variable period before diagnosis and challenge the perception of ovarian cancer as the "silent killer" [22].

Pelvic examination, many conditions that can affect women's health are often evaluated through pelvic examination. These conditions include malignant diseases, such as ovarian, uterine, vaginal, and cervical cancer; infectious diseases, such as bacterial vaginosis, candidiasis, genital warts, genital herpes, trichomoniasis, and pelvic inflammatory disease; and other benign conditions, such as cervical polyps, endometriosis, ovarian cysts, dysfunction of the pelvic wall and floor, and uterine fibroids. Pelvic examination is a common part of the physical examination; 44.2 million pelvic examinations were performed in the United States in 2012. Although it is a common part of the physical examination, it is unclear whether performing screening pelvic examinations in asymptomatic women has a significant effect on disease morbidity and mortality [23, 24].

Routine imaging tests, are noninvasive diagnostic imaging such as ultrasound performed with a transvaginal probe, computed tomography (CT), and magnetic resonance imaging (MRI), may help distinguish between benign and cancerous tumors. X-ray procedures are used if involvement of the colon or urinary tract is suspected. In women who have gastrointestinal symptoms, examination of the GI tract with upper and lower endoscopy is indicated to help rule out GI conditions and evaluate for bowel obstruction, and Positron Emission Tomography (PET) by radioactoring sugars to detect small group of cancer cells [21].

Laboratory investigations, included complete blood count (CBC), chemistry profile with a liver function tests (LFT) 
combined with alpha-fetoprotein (AFP), total serum proteins, and cancer antigen 125. Histopathological examination of ovarian tumors one of the most important method to differentiate between ovarian cancer types, used in staging and also in predicting the prognosis [25]. Tumor markers and Malignancy Indices, prospectively acquired evidence from the United Kingdom Collaborative Trial of Ovarian Cancer Screening Cancer (UKCTOCS) - with 46,237 women triaged using MMS in whom serial CA-125 measurements were interpreted via the risk of ovarian cancer algorithm (ROCA) has shown that screening by using ROCA doubles the number of screen-detected EOC compared with a fixed cut off of $35 \mathrm{IU} / \mathrm{ml}$ [19]. A Risk of Malignancy Index (RMI) was developed to estimate the probability of malignancy and the need to refer the patient to a tertiary hospital for optimal treatment. RMI is calculated by multiplying the menopausal status by the CA125 value and by certain sonographic features. Risk of Malignancy Index $(\mathrm{RMI})=\mathrm{M} \times \mathrm{CA} 125 \times \mathrm{U}$. RMI $>200=$ Suspicious for malignancy.

Biomarkers in the study, cancer biomarkers can be DNA, mRNA, proteins, metabolites, or processes such as apoptosis, angiogenesis or proliferation. The markers are produced either by the tumor itself or by other tissues, in response to the presence of cancer or other associated conditions, such as inflammation. Biomarkers can be found in a variety of fluids, tissues and cell lines. Tumor markers can be used for screening the general population, for differential diagnosis in symptomatic patients, and for clinical staging of cancer. Additionally, these markers can be used to estimate tumor volume, to evaluate response to treatment, to assess disease recurrence through monitoring, or as prognostic indicators of disease progression [26].

A number of different types and forms of tumor markers exist, these markers include hormones, as well as different functional subgroups of proteins such as enzymes, glycoproteins, oncofetal antigens and receptors. Furthermore, other changes in tumors, such as genetic mutations, amplifications or translocations, and changes in microarraygenerated profiles, $b$ are also forms of tumor markers. Only a few markers have entered routine use, and then only for a limited number of cancer types and clinical settings. In the majority of cases, the current markers are used in conjunction with imaging, biopsy and associated clinicopathological information before a clinical decision is made [26].

In this study, we aimed to evaluate the diagnostic performance of serum biomarker from patients presenting with ovarian cancer. we especially desired to investigate levels of Human epididymis protein4 (HE4).

Human epididymis protein4 (HE4) is a stable fourdisulphide core protein originally found lining the human epididymis. It is a whey acidic type of protein (WAP) comprising approximately 50 amino acids, including eight cysteines in a conserved arrangement. HE4 is thought to have a role in sperm maturation, yet its function is still somewhat unclear. Protease inhibiting action with significant binding to many seminal fluid proteases has been shown in protein-level studies [27]. The similarities with known leukocyte protease inhibitors in the WFDC protein family also suggest HE4 to have a role in natural immunity. The HE4 gene (WFDC2) resides on human chromosome 20q12-13.1. It has been found to be overexpressed in ovarian carcinomas, but not in normal ovaries, and being a small, secreted protein, it was introduced as a clinical marker for ovarian cancer [28].

High HE4 expression has been found in several normal epithelial tissues, such as trachea and salivary gland, and genital tracts in both genders outside of the testes and ovaries. In males, HE4 expression is the highest in the epithelial cells of the epidydimal and spermatic ducts, with low and sparse expression in the prostate. The highest expression in females is seen in endometrium, fallopian tubes, endocervical and Bartholin's glands, whereas little or no expression has been detected in myometrium, vulva and ovary. Ovarian cortical inclusion cysts that are lined by metaplastic Müllerian epithelium have shown higher HE4 expression levels than normal ovarian epithelium. [29]. The WFDC2 gene presents with variable levels of expression also in several distinct parts of the body such as colon, pancreas, kidney tubules, breast tissue, anterior pituitary, thyroid, lacrimal and eccrine glands.

Altogether five mRNA variants of the HE4 gene with different exonic arrangements have been identified as a result of variable splicing or utilization of alternative promoters. In both benign and malignant tissues, HE4-V0, -V1 and -V3 isoforms are most abundant, HE4-V0 depicting the prototype of the HE4 protein. The expression of all HE4 protein isoforms is significantly increased in endometrioid and papillary serous forms of endometrial cancer, whereas increased expression of HE4-V1,-V3 and -V4 variants present with a negative impact on survival of these patients (Jiang et al. 2013). Significant correlation of the protein isoform HE4-V3 and improved prognosis in adenocarcinoma of the lung has also been reported [30]. The vast majority of studies and methods detecting HE4 expression has been founded on the structure of HE4-V0 with no distinction between the isoforms. However, the14 Review of the Literature clinical relevance of the structural, and possibly functional, variation of the HE4 molecule appears limited, as HE4-V0 is present in 10-100-fold levels as compared to HE4-V1 and -V3 and in 100-1000-fold levels as compared to HE4-V2 and -V4 in benign tissues, similar finding being present in endometrial carcinoma.

Serum HE4 concentration can be measured using immunometric techniques; enzyme linked immunosorbent assay (EIA), chemiluminescent microparticle immunoassay (CMIA), electro chemiluminescence immunoassay (ECLIA) and chemiluminescent enzyme immunoassay (CLEIA). There is a commercial kit available for each technique [30]. Measurements with ELISA technique has been studied [28, 31], showing similar diagnostic specificity (94.4\%) and sensitivity $(86.6 \%$ in stage $\mathrm{I} / \mathrm{II}$ and $89 \%$ in stage III/IV ovarian cancer) as previous studies of serum measurements. The discrimination potential HE4 in differentiating ovarian cancer from benign ovarian tumors is considerable, detecting tumors with low malignant potential even better than serum 
HE4 [31]. The authors also reported no significant difference between HE4 concentrations analyzed from serum or EDTA plasma. Biological variation Smoking increases serum HE4 levels, yet the most significant non-malignant cause for elevated HE4 concentrations is renal insufficiency Ageing increases HE4 levels and age-dependent reference ranges have been suggested. In a meta-analysis by Moore et al. (2012) [32], the serum samples were pooled in age groups by decade and classified to pre- or postmenopausal according to age $(<45$ or $>55)$ or medical history. Clear increase of serum HE4 concentration by age was demonstrated, menopausal status not being an independent factor. The International Federation of Clinical Chemistry and Laboratory Medicine and the Clinical and Laboratory Standards Institute recommend determining cut points for laboratory values as upper confidence interval for the 95th percentile. In Asian population, the 95th percentile cutoff limit for a group of 2,182 women aged 20 - 65 years was detected by Park et al. 2012 [33] to be as low as $30.3 \mathrm{pM}$.

Clinical implications in gynecology the diagnosis of pelvic tumors comprises a combination of clinical examination, transvaginal ultrasound and serum tumor markers. For three decades, CA125 was the solely used marker for epithelial ovarian cancer. Now, within the past ten years, HE4 analysis has become commercially available, and it has been accepted for worldwide clinical use. In addition to the use in differential diagnosis of EOC, the usability of HE4 in EOC follow-up has been reported, with some evidence that HE4 increase could detect recurrence earlier than CA125 However, no studies on the effect of HE4 use in follow up in patient survival have been published, taken into consideration the clinical effects of the randomized controlled trial by Rustin and colleagues (2010).

They presented no survival benefit from early treatment of EOC recurrence based on early CA125 rise as compared to starting treatment only when symptoms occur. In contrast to CA125, elevated serum HE4 concentrations have also been reported to correlate with platinum resistance at the time of the third chemotherapy cycle, suggesting that HE4 might be valuable in treatment planning HE4 EIA analysis was approved by the American Food and Drug Administration (www.fda.gov) for monitoring patients with ovarian cancer and in 2011 it was approved together with CA125 analysis to estimate the risk of ovarian malignancy [33].

Different subtypes of ovarian cancer, however, seem to require a different approach. In addition to serous ovarian carcinoma, most clear-cell and endometrioid carcinomas express HE4 in immune histochemical staining. However, HE4 is expressed only rarely in ovarian mucinous, germ cell and sex cord stromal tumors. These less common types of ovarian cancer often present a diagnostic challenge, and the development of targeted specific tumor markers could be very beneficial in clinical work. In some studies, HE4 has been identified as the most sensitive marker in stage I EOC [32]. Nevertheless, a recent, large study published opposite results Review of the Literature questioning the superiority of HE4 in early diagnosis [34]. neither perform exclusively in diagnosing the early stages non-ovarian cancers HE4 can be elevated also in non-gynecological cancers. A small study done by Park et al. 2011 [35], of 31 women with a nongynecological (mostly breast, some gastrointestinal and pancreatic) and nine with non-ovarian gynecological (cervical or endometrial) cancer detected a difference between the non-gynecological cancers and the healthy control group ( $84 \mathrm{pM}$ vs. $48 \mathrm{pM}, \mathrm{p}=0.0004$ ), whereas the slight elevation of HE4 levels in non-ovarian gynecological cancers was not significant compared to healthy controls (62 pM vs. 48 pM) [35]. Other neoplasms that show strong HE4 expression are adenocarcinomas of the lung and certain tumors of the salivary gland, thyroid, breast and pancreas and transitional cell tumors of the bladder. Some HE4 upregulation has also been found in gastric and pancreatic carcinomas, however, showing no correlation with patient outcome, and only of modest diagnostic value, thus far with no clinical advantage.

Serum HE4 concentrations have been found significantly elevated in primary adenocarcinoma of the lung compared to healthy individuals with potential as a diagnostic and prognostic marker for lung cancer. Single studies showing serum HE4 increase in pancreatic cancer and transitional cell cancer of the bladder have also been published [36]. The best combination of markers was CA125 with HE4 as expected, however, in Marianne Hallamaa 2017 [36] study they were not found superior to CA125 only in EOC vs. healthy controls, all stages and histology included.

\section{Materials and Methods}

Materials, a total of 90 Sudanese ladies age range (16-80) years old attending Gynological Oncology clinics in Omdurman Military hospitals - Khartoum state from May 2015 to December 2016 was included in the study. The study was analytical comparative cross-sectional study. The sample population was divided into two main groups; study group including 53 (58.8\%) Ovarian cancer patient with an age of 16 to 80 years, and control group including 37 (31.2\%) aged match apparently healthy individuals according to the study Inclusion criteria including of Sudanese women diagnosed with primary ovarian cancer and excluded any women diagnosed with other cancer types rather than ovarian cancer.

History and background data were collected from participants using verbal interviews and pre-designed questionnaire. Clinical presentation includes an enlarged ovary on a pelvic exam, ascites, and histopathological examinations to regulate the tumor type, ovarian cancer type, and staging of the disease, then followed by metastatic status of cancer. Five to ten $\mathrm{ml}$ blood samples were collected from each participant; sera were separated, and then stored at $20^{\circ} \mathrm{C}$ for subsequent testing. Biomarker Human epididymis protein 4 (HE4) were performed to all serum samples to determine the concentration, by Enzyme-Linked Immunosorbent Assay techniques (CUSABIO ELISA Kits).

Written and informed consents were obtained from all ovarian cancer patients prior to involvement in the study. 
Ethical release to proceed in the study was obtained from the ethical committee of the Faculty of Medical Laboratory Sciences at Al Neelain University.

Methods, Enzyme-Linked Immunosorbent Assay for the quantitative invitro diagnostic measurement of Human epididymis protein 4 (HE4).

Principle, this assay employs the quantitative sandwich enzyme immunoassay technique. Antibody specific for HE4 has been pre-coated onto a microplate. Standards and samples are pipetted into the wells with a Horseradish Peroxidase (HRP) conjugated antibody specific for HE4. Following a wash to remove any unbound reagent, a substrate solution is added to the wells and color develops in proportion to the amount of HE4 bound in the initial step. The color development is stopped and the intensity of the color is measured.

Preparation of Reagents and sample, allowed all reagents and required number of strips to reach room temperature (18$25^{\circ} \mathrm{C}$ ) prior to use, distilled water was used to make reagents preparation. Wash Buffer (1x) after warmed up to room temperature mixed gently until the crystals completely dissolved. $30 \mathrm{ml}$ of Wash Buffer Concentrate $(20 \mathrm{x})$ was diluted into distilled water and $600 \mathrm{ml}$ prepared of Wash Buffer $(1 \mathrm{x})$, thawed samples inverted several times prior to testing.

Assayed Procedure, secured the desired number of Microtiter wells in the frame holder, dispensed $25 \mu \mathrm{L}$ of each Standard, Control and samples with new disposable tips into appropriate wells, $75 \mu \mathrm{l}$ of HRP-conjugate was added to each well. Mixed well and then incubated for 1 hour at $37^{\circ} \mathrm{C}$. Aspirated each well and washed four times for a total of five washes, then filling each well with Wash Buffer $(300 \mu l)$ by multi-channel pipette, and let it stand for 20 seconds, complete removal of liquid at each step was performed any remaining was removed. Added $50 \mu 1$ of Substrate A and $50 \mu 1$ of Substrate B to each well, mixed well, incubated for 15 minutes at $37^{\circ} \mathrm{C}$. the plate Kept in the dark. Added $50 \mu \mathrm{l}$ of Stop Solution to each well, the plate taped gently to ensure thorough mixing. Finally, the optical density of each well was determined within 10 minutes, using a microplate reader set to $450 \mathrm{~nm}$.

Calculations of Results, by using automated method, in this method the results have been calculated automatically using a 4 PL (4 Parameter Logistics) curve fit. 4 Parameter Logistics is the preferred method. The concentrations of the samples were read directly from this standard curve.

Detection range $75 \mathrm{pmol} / \mathrm{L}-1000 \mathrm{pmol} / \mathrm{L}$

Quality control, the controls were running with each calibration curve for the five parameters, a statistically significant number of controls were assayed to establish mean values and acceptable ranges to assure proper performance. Using control sera at both normal and pathological levels. The checking of the following technical areas: Pipetting and timing devices; photometer, expiration dates of reagents, storage and incubation conditions, aspiration and washing methods were done.

Statistical analysis, Raw data were entered into a spread sheet of SPSS statistical package program, data were rearranged as appropriate. Descriptive analysis was performed to all study variables.

Data was analyzed using SPSS version 21. The results expressed as mean, standard deviation, median, frequency and percentage. Descriptive statistic was done to obtained the frequencies and percentages of the study variables and clinical data. Independent-sample T-test was demonstrated to compare the mean concentration of OVC biomarker parameter $\mathrm{HE} 4$ in OVC cancer versus healthy individual (control groups). One-way ANOVA was used to mean concentration of OVC biomarker parameter HE4, across the OVC stages. Graphs were done using Microsoft excel and Graph Pad Prism version 6. P-value $\leq 0.05$ was considering as significantly difference. All statistics tests were done in confidence interval $95 \%$.

\section{Results}

Clinical Results, ninety Sudanese females were enrolled in this study. They were distributed into two groups; Study group including 53 newly diagnosed ovarian cancer patients age ranged (16-80) years old, and Control group including 37 age match apparently healthy individuals. Study group include $32 \%$ in the reproductive age and about $68 \%$ elderly female which shown in figure 1.

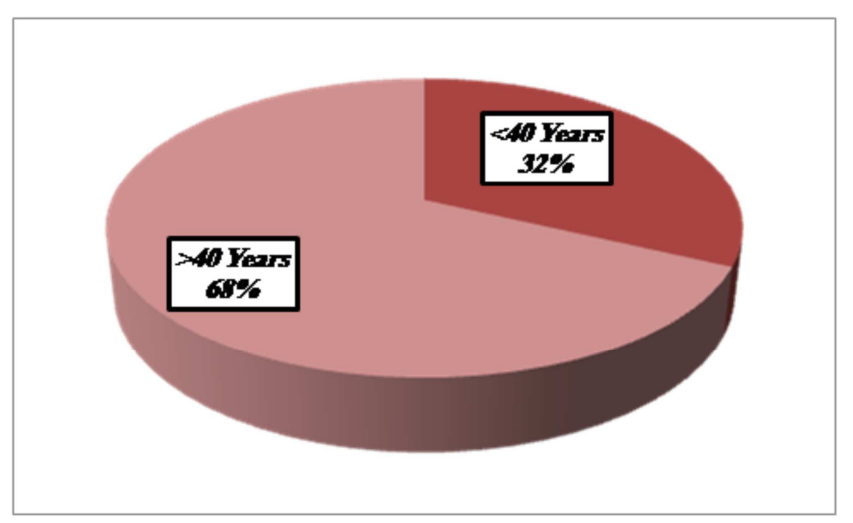

Figure 1. Age percentage among ovarian cancer patients.

The frequency and percentage of signs and symptoms (table 1) shown that $79.0 \%$ from the study group suffering from abdominal bloating, $62 \%$ loss of appetite, $68 \%$ urinate more frequent, $57 \%$ irregular bowel movement, 70\% presented with increased abdominal size, about $85 \%$ with abdominal pain, all study group deny history of ovarian cancer in their families, only $13 \%$ of ovarian cancer patient using pills as contraceptive as well as $4 \%$ hormonal therapy consumption, 8\% Caesarean as gynecological surgery. As well as about $51 \%$ of the study group were para and multiparity compared with $49 \%$ were nulliparous shown in figure 3. And $45 \%$ of this study group suffering from asities when clinical examination done and confirmed by ultrasonography also signify percentage of the left (Lt), right (Rt), and Bilateral ovarian mass as $19 \%, 34 \%$, and $47 \%$ respectively shown in figure 2. 
Table 1. Frequency and percentage of common symptoms among ovarian cancer patients.

\begin{tabular}{|c|c|c|}
\hline Variables & Frequency & Percentage \\
\hline \multicolumn{3}{|c|}{ Abdominal bloating } \\
\hline Present & 42 & $79.0 \%$ \\
\hline No & 11 & $21.0 \%$ \\
\hline \multicolumn{3}{|c|}{ Loss of appetite } \\
\hline Present & 33 & $62.0 \%$ \\
\hline No & 20 & $38.0 \%$ \\
\hline \multicolumn{3}{|c|}{ Urinate more frequent } \\
\hline Present & 36 & $68.0 \%$ \\
\hline No & 17 & $32.0 \%$ \\
\hline \multicolumn{3}{|c|}{ Irregular bowel movement } \\
\hline Present & 30 & $57.0 \%$ \\
\hline No & 23 & $43.0 \%$ \\
\hline \multicolumn{3}{|c|}{ Increased abdominal size } \\
\hline Present & 37 & $70.0 \%$ \\
\hline No & 16 & $30.0 \%$ \\
\hline \multicolumn{3}{|c|}{ Abdominal pain } \\
\hline Present & 45 & $85.0 \%$ \\
\hline No & 8 & $15.0 \%$ \\
\hline \multicolumn{3}{|c|}{ History of ovarian cancer } \\
\hline Present & 0.00 & $0.00 \%$ \\
\hline No & 53 & $100.0 \%$ \\
\hline \multicolumn{3}{|c|}{ Using of contraceptive } \\
\hline Pills & 7 & $13.0 \%$ \\
\hline No & 46 & 87.0 \\
\hline \multicolumn{3}{|c|}{ Hormonal therapy } \\
\hline Estrogen & 2 & $4.0 \%$ \\
\hline No & 51 & $96.0 \%$ \\
\hline \multicolumn{3}{|c|}{ Gynecological surgery } \\
\hline Caesarean & 4 & $8.0 \%$ \\
\hline No & 49 & $92.0 \%$ \\
\hline \multicolumn{3}{|l|}{ Asities } \\
\hline Present & 24 & $45.0 \%$ \\
\hline No & 29 & $55.0 \%$ \\
\hline
\end{tabular}

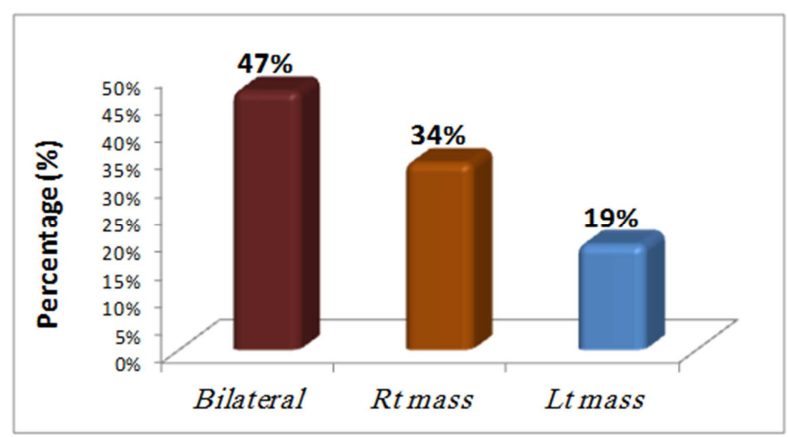

Figure 2. Ovarian mass among ovarian cancer patients.

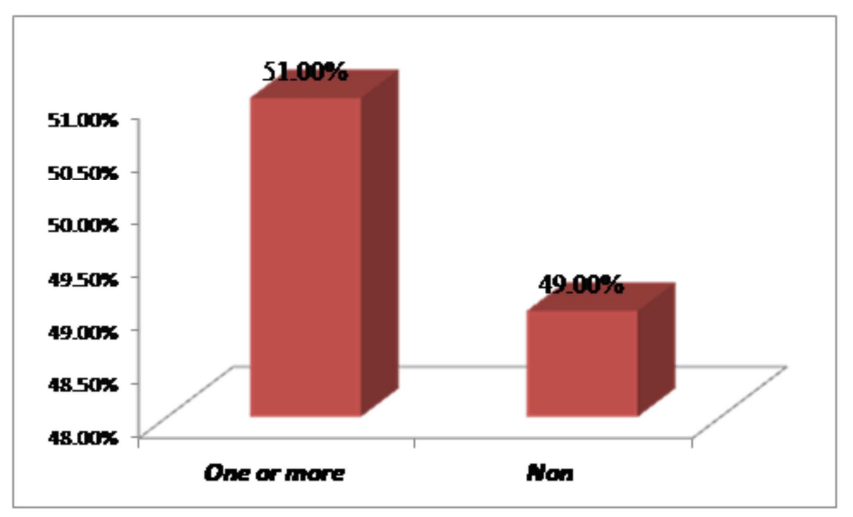

Figure 3. Parity percentage among ovarian cancer patients.

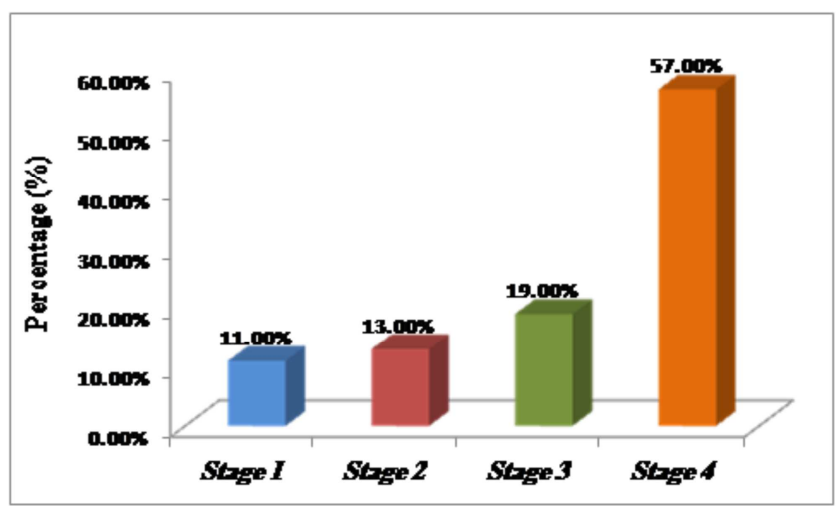

Figure 4. ovarian cancer stages among study group.

Laboratory Results, Histopathological results, the present study showed $97 \%$ of the ovarian cancer were epithelial cell origin and only $3 \%$ were germ cell origin. Staging of ovarian cancer among study group grading from stage 1, 2, 3 and, 4 were $11 \%, 13 \%, 19 \%$ and $57 \%$ respectively showed in figure 4.

Serum biomarker results, the present study showed differences of serum biomarker human epididymis protein 4 (HE4), levels among ovarian cancer and control individuals Findings varied between these study groups in the levels of HE4. For instance, level detected among participants in the study group showed increased serum level and normal level among control group. Table 2 .

Results of the serum HE4 showed variation in the concentrations among both Para/ multi parity and Nulliparous sub groups of ovarian cancer patients table 3 .

Table 2. Serum HE4 among study and control group.

\begin{tabular}{lllll}
\hline Parameter & Case $($ Mean \pm SD) & Median & Control (Mean \pm SD) & Median \\
\hline HE4 $(\mathrm{pmol} / \mathrm{L})$ & $484.65 \pm 58.88$ & 250.00 & $42.66 \pm 6.29$ & 50.00 \\
\hline
\end{tabular}

Table 3. Serum HE4 among parity sub grouping.

\begin{tabular}{llll}
\hline Parameter & Para/ multi parity (Mean \pm SD) & Nulliparous (Mean \pm SD) & P-value \\
\hline HE4 $(\mathrm{pmol} / \mathrm{L})$ & $550.61 \pm 93.81$ & $416.15 \pm 69.48$ & 0.009 \\
\hline
\end{tabular}

Table 4. Serum biomarkers among ovarian cancer stages.

\begin{tabular}{llllll}
\hline Parameter & Stage 1 (Mean \pm SD) & Stage 2 (Mean \pm SD) & Stage 3 (Mean \pm SD) & Stage 4 (Mean \pm SD) & P-value \\
\hline HE4 $(\mathrm{pmol} / \mathrm{L})$ & $354.0 \pm 115.4$ & $535.0 \pm 156.1$ & $499.0 \pm 219.1$ & $513.6 \pm 180.7$ \\
\hline
\end{tabular}


HE4 mean concentration was $484.65 \mathrm{pmol} / \mathrm{L}$ in the study group, and $42.66 \mathrm{pmol} / \mathrm{L}$ in the control group shown significant difference with p-value 0.0001 along with mean concentration of Para/ multi parity and Nulliparous sub groups of ovarian cancer patients were $550.61 \mathrm{pmol} / \mathrm{L}$, $416.15 \mathrm{pmol} / \mathrm{L}$ respectively shown significant difference with $\mathrm{P}$-value $=0.009$ table 3 . Mean concentrations of this marker among cancer stages 1, 2, 3, and 4 shown 354.0, 535.0,499.0, and 513.6 respectively which shown insignificant difference with p-values $(0.771)$ demonstrate in table 4 and Figure 5. The sensitivity $98 \%$, Specificity $98 \%$, Positive predictive value, and $92 \%$ Negative predictive value $86 \%$ shown on table 5 .

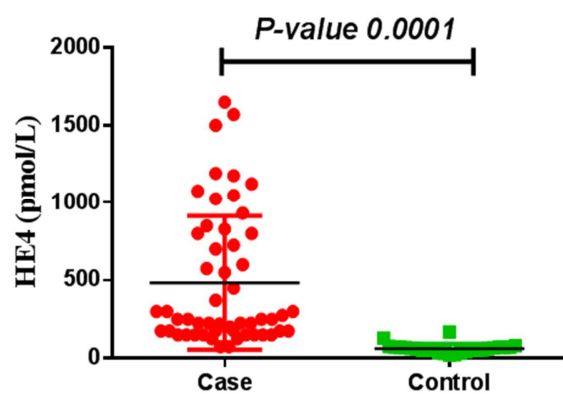

Figure 5. Mean concentration of HE4 among ovarian cancer patient

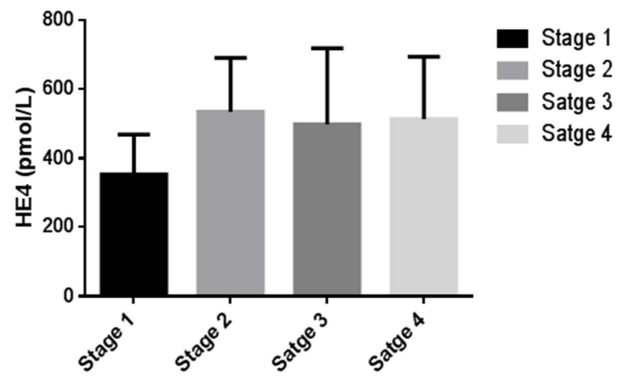

Figure 6. Mean concentration of HE4 among ovarian cancer stages.

Table 5. Sensitivity, Specificity, and predictive values for HE4.

\begin{tabular}{llllll}
\hline Variables & A. U.C & Sensitivity & Specificity & P. P. Value & N. P. Value \\
\hline $\begin{array}{l}\text { HE4 } \\
(\mathrm{pmol} / \mathrm{L})\end{array}$ & 0.990 & $98 \%$ & $98 \%$ & $92 \%$ & $86 \%$ \\
\hline
\end{tabular}

\section{Discussion}

Amongst all gynecological cancers, ovarian cancer is the most lethal malignancy worldwide, aggressive local invasion and the lack of sensitive early screening methods, poses the most difficult in early diagnosis, furthermore, its high mortality rate has made it one of the most investigated fields in gynecological oncology during this year in USA ovarian cancer ranks fifth in cancer deaths among women [1], a woman's risk of getting ovarian cancer during her lifetime is about 1 in 75, her lifetime chance of dying from ovarian cancer is about 1 in 100 according to Ovarian Cancer Treatment Statistics and Results of Cancer Treatment Centers of America 2012 [65].

Even though OVC mainly develops in older women there is younger age range were reported in review study done by Wisal etal 2017 [66], among Sudanese ovarian cancer patient which agree with our study because there were thirty tow percent within reproductive age.

The results of the present work affirm that, around fifty seven percent of all ovarian cancers included in this study were diagnosed at an advanced stage and only eleven percent in early stage. Then the five-year survival rate for patients with clinically advanced ovarian cancer is only fifteen to twenty percent, in striking contrast to a five-year survival rate of over ninety percent for patients with stage I disease [67].

In this study, we found the common symptoms among OVC patients involved in the clinical presentation are abdominal bloating, pelvic pain, abdominal pain, increase abdominal size vaginal discharge with the highest frequent, and vaginal bleeding with low frequency, these findings similar to cancer facts and figures published in 2017 by American cancer society [20]. Ultrasonography as noninvasive diagnostic test in women with pelvic, bilateral, and ascites are helpful in predicting the likelihood that mass is malignant [24]. Ovarian tumors were unilateral in 53\% of cases and bilateral in $47 \%$ with right side predominance This also chimes with the findings of Jyothi Kancherla etal [25].

Histopathological distribution in our study group is similar to many published works [37] [39], ovarian epithelial cell being the most common and followed by Germ cell, which present in different age ranges included in this study, Germ cell neoplasm present among younger age in the study group, present study findings are broadly similar to Kancherla etal [25] who reported that surface epithelial tumors were most common (80\%) followed by germ cell tumors $(16 \%)$.

In the present study, HE4 is an epididymis protein over expression was identified in patients suffering from all ovarian cancer stages which offers better sensitivity $98 \%$ and specificity $98 \%$ than CA125 as well as PPV 92\% and NPV86\% This suggests similar to NCCN Guidelines Version 2013 [21]. Hellstrom and colleagues showed that secreted HE4 was detected in high levels in the serum of ovarian this group found that measurement of HE4 showed sensitivity and specificity comparable to that of CA125 for differentiating women with ovarian cancer from normal controls [25]. Høgdall, Estrid and colleagues for the first time in Denmark presented a single marker, HE4, with a higher diagnostic prediction than the golden standard CA 125 [40]. Drescher, at Hutchinson Cancer Research Center, USA found that HE4 performance better than CA125 serum levels which get same results with our study $[41,42]$.

Unfortunately, most studies of tumor markers are highly variable, not only in their methods of marker detection, but also in design and patient selection. Interpatient heterogeneity and intratumor heterogeneity are important confounding factors. In addition, the danger of bias and the problems of overfitting the data, as well as issues relating to the handling and storing of clinical specimens, are vital factors that need consideration before a study is conducted. New tumor marker tests - single or multiparametric - must, 
therefore, undergo rigorous validation in order that their clinical value can be assessed.

Reaching to scientific evidence in this study, the HE4 biomarker yield results that fall within the normal range in the control group. In contrast, increasing results were obtained in the ovarian cancer study group. For instance, Human epididymis protein 4 (HE4) level significantly increased in all ovarian cancer patient included in this study.

\section{Conclusion}

By the end of this study, concludes that epithelial ovarian cancer is the most common followed by germ cell tumors. Serum level of Human epididymis protein 4 (HE4) within the reference range in the control group. In contrast, increasing serum level of were obtained in the ovarian cancer patients, A general agreement that a combination of multiple biomarkers may increase diagnostic sensitivity and specificity over use of individual markers. The results of previous studies [42] study confirmed that, by combining CA125, and $\mathrm{He} 4$ measurements, we can improve the diagnostics performance for OC. HE4 is a relatively stable serum biomarker for ovarian cancer with a higher diagnostic prediction than the golden standard CA 125.

\section{Recommendations}

Stablishing of HE4 as ovarian biomarker with a golden standard CA125, and pointing to a rationale for further research assessing potential clinical usefulness. Less invasive and Cost- effective methods for studied serum biomarkers analysis most being developed in combination with modern information technology, such as a possibility of urine sampling for HE4 measurement and further assay interpretation with a mobile application.

\section{References}

[1] National Comprehensive Cancer Network (NCCN). Ovarian Cancer Including Fallopian Tube Cancer and Primary Peritoneal Cancer. Available from: http://www.nccn.org/professionals/physician_gls/pdf/ovarian. pdf. 2016. USA.

[2] Schwartz PE. Current diagnosis and treatment modalities for ovarian cancer. Cancer Treat Res 2002; 107: 99-118.

[3] Kurman RJ, Carcangiu ML, Herrington CS, Young RH. WHO classification of tumors of female reproductive organs 4th ed. Lyon: International Agency for Research on Cancer; 2014.

[4] Prat J. Ovarian carcinomas: five distinct diseases with different origins, genetic alterations, and clinicopathological features. Virchows Arch 2012; 460: 237-49.

[5] Jaime Prat. Staging classification for cancer of the ovary, fallopian tube, and peritoneum. Int J Gynecol Obstet 2014; 124 (1): 1-5. JGynecol Oncol Vol. 26, No. 2: 87-89 http://dx.doi.org /10.3802/jgo.2015.26.2.87
[6] Shahrazad Ehdaivand, etal. 2016, WHO classification of Ovarian Neoplasms, Pathology Outlines.com, Inc.

[7] Yao Chen, Suihai Wang, Tiancai Liu, Yingsong Wu, Ji-Liang $\mathrm{Li}$, and Ming $\mathrm{Li}, 2016$. WAP four-disulfide core domain protein 2 gene (WFDC2) is a target of estrogen in ovarian cancer cells. Journal of Ovarian Research 2016; 9: 10.

[8] American Joint Committee on Cancer. Ovary and Primary Peritoneal Carcinoma. In: AJCC Cancer Staging Manual. 7th ed. New York: Springer; 2010: 419-428.

[9] American Cancer Society. Cancer Facts and Figures 2016. Atlanta, GA: American Cancer Society; 2016.

[10] Melinda M. Protani, Christina M. Nagle, and Penelope M. Webb. Obesity and Ovarian Cancer Survival: A Systematic Review and Meta-analysis, www.aacrjournals.org, 2012; DOI: 10.1158/1940-6207.CAPR-12-0048.

[11] Valerie McGuire, Patricia Hartge, Linda M. Liao, Rashmi Sinha, Leslie Bernstein, Alison J. Canchola, Garnet L. Anderson, Marcia L. Stefanick, Alice S. Whittemore. Parity and Oral Contraceptive Use in Relation to Ovarian Cancer Risk in Older Women. 2016; DOI: 10.1158/1055-9965.EPI16-0011.

[12] Chowdhury R, Sinha B, Sankar MJ, et al. Breastfeeding and maternal health outcomes: a systematic review and metaanalysis. Acta Paediatr. 2015; 104 (467): 96-113.

[13] Alison Volpe Holmes, Heather G. Jones, Brock C. Christensen, Breastfeeding and Cancer Prevention, April, 2017. NH Comprehensive Cancer Collaboration in partnership with Dartmouth-Hitchcock Norris Cotton Cancer Center and its NCI National Outreach Network Community Health Educator Site.

[14] Muhammad Zahid, Cheryl L. Beseler, James B. Hall, Tricia LeVan, Ercole L. Cavalieri and Eleanor G. Rogan. Unbalanced estrogen metabolism in ovarian cancer, International Journal of Cancer. 25 January 2014. DOI: $10.1002 / \mathrm{ijc} .28565$.

[15] Sami Azrak. Hereditary Ovarian Cancer and Germline Mutations: Review Article. Journal of Genetics and Genetic Engineering Volume 1, Issue 1, 2017, PP 31-42.

[16] American collage of Obstetrician and Gynecologist, fact sheet in BRCA1 and BRCA2 mutations. Oct 2017.

[17] Francis S. Collins. Study reveals genomic similarities between breast cancer and ovarian cancers. National Cancer Institute (NCI) and the National Human Genome Research Institute (NHGRI), both part of NIH. Sept. 23, 2012.

[18] Jacobs IJ, Menon U. Progress and challenges in screening for early detection of ovarian cancer. MolCell Proteomics 2004; 3 : $355^{\wedge} 66$.

[19] Hristina Fotopoulou, Marcia Hall, Derek Cruickshank, Hani Gabra, Raji Ganesan, Cathy Hughes, Sean Kehoe, Jonathan Ledermann, Jo Morrison, Raj Naik, Phil Rolland, Sudha Sundar. British Gynaecological Cancer Society (BGCS) Epithelial Ovarian /Fallopian Tube / Primary Peritoneal Cancer. 2014; 1-48. Guidelines: https://www.rcog.org.uk/en/guidelinesresearchservices/guidelines/clinical-governance-advice-1a/.

[20] American cancer society, cancer facts and figures 2017. special section: Rare cancers in adults. Atlanta 2017. https://www. American cancer society.com. 
[21] National Comprehensive Cancer Network. NCCN Guidelines Version 4. Hereditary Breast and/or Ovarian Cancer Syndrome. 2013. USA.

[22] Network SIG. Management of epithelial ovarian cancer. SIGN Publication No 135. Edinburg: SIGN; 2013.

[23] Health Resources \& Services Administration. Women's preventive services guidelines. https://www.hrsa.gov/womensguidelines2016/index.html. Accessed January 17, 2017.

[24] American Family Physician. screening for Gynecologic Conditions with Pelvic Examination: Recommendation Statement, August 15, 2017. Volume 96, Number 4 www.aafp.org/afp.

[25] Jyothi Kancherla, Raghu Kalahasti, K P A Chandra Sekhar, Srikanth Babu Yarlagadda, S Parimala Devi 2017. Histomorphological Study of Ovarian Tumors: An Institutional Experience of 2 Years. International Journal of Scientific Study | June 2017 | Vol 5 | Issue 3.

[26] Vathany Kulasingam and Eleftherios P Diamandis. Strategies for discovering novel cancer biomarkers through utilization of emerging technologies nature clinical practice oncology oct 2008, vol 5, no 10 .

[27] Chhikara, N., M. Saraswat, A. K. Tomar, S. Dey, S. Singh, and S. Yadav. Human epididymis protein-4 (HE-4): a novel crossclass protease inhibitor." PLoS One 2012; 7 (11): e47672.

[28] Hellstrom, I., P. J. Heagerty, E. M. Swisher, P. Liu, J. Jaffar, K. Agnew, and K. E. Hellstrom."Detection of the HE4 protein in urine as a biomarker for ovarian neoplasms. Cancer Lett. 2010 Oct 1; 296 (1): 43-8. Cancer.

[29] Georgakopoulos, P., S. Mehmood, A. Akalin, and K. R. Shroyer. Immunohistochemical localization of HE4 in benign, borderline, and malignant lesions of the ovary." Int J Gynecol Pathol 2012; 31 (6): 517-23.

[30] Tokuishi, K., S. Yamashita, K. Ohbo, and K. Kawahara. Splice variant HE4- V3 expression is associated with favorable prognosis in pulmonary adenocarcinoma. Tumour. 2012 Feb; 33 (1): 103-9.

[31] Liao, J. B., Y. Y. Yip, E. M. Swisher, K. Agnew, K. E. Hellstrom, and I. Hellstrom. Detection of the HE4 protein in urine as a biomarker for ovarian neoplasms: Clinical correlates." Gynecol Oncol 2015; 137 (3): 430-5.

[32] Moore, R. G., M. C. Miller, E. E. Eklund, K. H. Lu, R. C. Bast, and G. Lambert-Messerlian. Serum levels of the ovarian cancer biomarker HE4 are decreased in pregnancy and increase with age." Am J Obstet Gynecol 2012; 206 (4): 349. e1-7.

[33] Park, Y., Y. Kim, E. Y. Lee, J. H. Lee, and H. S. Kim. Reference ranges for HE4 and CA125 in a large Asian population by automated assays and diagnostic performances for ovarian cancer." Int J Cancer 2012; 130 (5): 1136-44.

[34] Terry, K. L., H. Schock, R. T. Fortner, etal 2016. "A prospective evaluation of early detection biomarkers for ovarian cancer in the European EPIC cohort." Clin Cancer Res. 22 (18): 4664-75.

[35] Park, Y., J. H. Lee, D. J. Hong, E. Y. Lee, and H. S. Kim. Diagnostic performances of HE4 and CA125 for the detection of ovarian cancer from patients with various gynecologic and non gynecologic diseases." Clin Biochem 2011; 44 (10-11): 884-8.

[36] Marianne Hallamaa. Overian cancer marker HE4 in hormonerelated gynecological conditions and diagnosis of overian granulosa cell tumors turn ylopiston julkaisuja- Annales universitis Turkuensissarj. - ser. D osa - tom. 1278 | Medica Odontologica 2017 | Turku 201.

[37] US Preventive Services Task Force. Screening for Ovarian cancer. Accessed November 10, 2014 http://www.uspreventiveservicestaskforce.org/uspstf/uspsovar. htm.

[38] Skates S. OcS: development of the risk of ovarian cancer algorithm (ROCA) and ROCA screening trials. Int J Gynecol Cancer. 2012; 22 (suppl 1): S24-S26.

[39] American college of Obstetricians and Gynecologists. Practice bulletin no. 83: management of Adnexal masses. Washington, dc: American college of Obstetricians and Gynecologists; 2007.

[40] Høgdall, Estrid, Nedergaard, Karlsen. Diagnostic value of HE4, CA125 and the roma index in ovarian cancer. 2011, Denmark.

[41] Drescher, Urban N, Hartge. Potential role of HE4 in multimodal screening for epithelial ovarian cancer 2011, USA.

[42] Rimaz A. Gurashi, Moawia E. Hummeida, F. G. Abdelaziz. Diagnostic Value of Serum Cancer Antigen125 in Ovarian Cancer Patients. International Journal of Development Research Vol. 08, Issue, 01, pp. 18644-18650, January, 2018. 